\title{
Risk Detection of Malignant Tumors in Mammograms using Unconventional Computing
}

\author{
Jesús Emmanuel Velázquez-Cruz¹, Itzamá López-Yáñez², Amadeo José Argüelles-Cruz¹, \\ and Cornelio Yáñez-Márquez ${ }^{1}$ \\ ${ }^{1}$ Instituto Politécnico Nacional, Centro de Investigación en Computación, Mexico City, Mexico \\ jvelazquez_a13@sagitario.cic.ipn.mx, jamadeo@cic.ipn.mx, \\ cyanez@cic.ipn.mx \\ ${ }^{2}$ Instituto Politécnico Nacional, Centro de Innovación y Desarrollo Tecnológico en Cómputo, \\ Mexico City, Mexico \\ ilopezy@ipn.mx
}

\begin{abstract}
In this paper, we propose the use of Alpha-Beta associative approach as an Unconventional Computing method in the pre-diagnosis of malignant tumors of breast cancer, obtaining an accurate result in a simple way; trying to avoid invasive diagnostic methods like biopsies, as far as possible.

This proposal provides for the Alpha-Beta Support Vector Associative Machine created in 2008 and tested for classification of binary images. The results show that the classification model to detect malignancy is very competitive compared to others of the best known classification methods, having an accuracy of $81.85 \%$.
\end{abstract}

Keywords: Breast cancer, binary images, Alpha-Beta Support Vector Associative Machine, unconventional computing, Otsu method.

\section{Introduction}

In this work we use the Alpha-Beta associative approach to pattern recognition by means of Alpha-Beta Support Vector Associative Machine ( $\alpha-\beta$ SVAM) to detect the risk that a patient has a malignant type of breast cancer starting from mammograms classification.

Cancer is a malignant tumor generally characterized by loss of control of growth, development and cell division capable to reproduce metastasis or tumor spread. In Mexican women, from 2006, breast carcinoma has become the leading cause of death by cancer. According to the Instituto Nacional de Estadística y Geografia (INEGI) in 2009 occurred 4,964 deaths attributable to breast cancer, which accounted for $2 \%$ of all women's death [1]. The Instituto Nacional de Salud Pública (INSP) has shown that the review by mammography reduces women's mortality by breast cancer in $16 \%$ with appropriate treatment along 5 years [2]. The Clinical Practice Guideline for the Diagnosis and Treatment of 
Breast Cancer by Mexican Ministry of Health, states all patients should have a clinical examination, establishing the following diagnostic procedures [3]:

* Imaging studies (mammography or breast ultrasound) and Magnetic Resonance Imaging (MRI) in special cases.

* Biopsies: fine needle, cutting needle biopsy.

* Histopathology.

* Additional studies (liver biometrics, clotting times, blood chemistry, bone scan, etc.).

However, biopsies of any type can represent unpleasant side effects for women who undergo this procedure, and $10 \%$ have serious problems with bruising, haemorrhage and infections in the cut or invasion areas [4].

Therefore, research has been conducted around the world, including the European Conference on Artificial Intelligence in Medicine (AIME) which work with technical topics of data mining, pattern recognition, image processing and natural language processing, and others [5].

Different techniques have been implemented to prevent the increase of this disease in women and, currently, also in men, we need to educate them and create a culture of prevention, [6-7], or by using Artificial Intelligence (AI) techniques to make an effective prediagnosis avoiding subjecting patients to invasive methods such as biopsies as far as possible.

In medicine different Intelligent Computing techniques are used [8-9] and within them, the methods that have shown better results are neural networks, fuzzy logic and genetic algorithms, of which the combination between fuzzy logic and neural networks is the most widely used method [10]. In this sense, the applications of Intelligent Computing techniques have been reared towards different particular problems, such as the use of Artificial Neural Networks (ANN) to model the behavior of schizophrenia [11], also they are used to diagnose urological diseases [12] and determining the seminal quality in fertility tests [13]. The Naïve Bayesian method (NB) was implemented for the celiac disease diagnosis (intolerance to wheat, barley, rye and oats) [14]. Also there is the implementation of a particular case of ANN, such as the Multilayer Perceptron (MLP) for purposes of diagnosis in cerebral lesions [15] or identifying periventricular leukomalacia, which is a brain damage in children that generates holes [16].

Some of the methods that have been tested with good results using data from cancer patients are:

*Genetic algorithms: [17].

*k-nearest neighbors (kNN): [18].

*Fuzzy Logic: [19].

*Support vector machines (SVM): [20-25].

*Artificial neural networks (ANN): [26-29].

*Semi-supervised learning (SSL): [30].

*Naïve Bayes (NB): [31]. 
There is an approach that despite not having many years of development, it has shown competitive results in different areas of knowledge, the associative approach. This approach has its roots at two pattern recognition models: the Alpha-Beta Associative Memories [32] ---whose $\alpha$ and $\beta$ operators are the bases for almost every subsequent associative model--- and the Hybrid Associative Classifier [33] ---which is the first associative model designed specifically for pattern classification--- which combines two important models of associative memories to generate an efficient classifier. However, this approach has seldom been applied for classification of medical images. One successful antecedent in such topic is the model of the $\alpha-\beta$ SVAM [34]; designed for classification of binary images (black and white) as patterns, tested with image set of handwritten letters and numbers.

\section{Methods}

In this section the necessary techniques to get the best information from medical imaging (mammographies) are described, which will be classification patterns. One of this proposal's most important parts is the image segmentation by binarization, because $\alpha-\beta$ SVAM requires binary images to recover or classify them, this is presenting a dynamic thresholding technique. After that, the $\alpha-\beta$ SVAM algorithm is shown, which will be explain to understand how it classify an image like a pattern.

\subsection{Image Binarization by Thresholding and the Otsu Method}

Binarization is an image processing technique which reduces information in a two values digital image: 0 (black) and 255 (white).

Thresholding is a segmentation technique used to binarize images when there is a clear difference between the objects to be extracted and the background in an image.

Most thresholding techniques are based on the one-dimensional histogram statistics. There are parametric procedures where the gray levels distribution of an object class leads to find appropriate thresholds; also there are nonparametric methods where the thresholds are obtained optimally according to some criterion.

Otsu method is a nonparametric procedure that selects the optimum threshold by maximizing the variance between classes using an exhaustive search.

An image is a two dimensional function for the gray level intensity what contains $N$ pixels whose gray levels are between 1 and $\boldsymbol{L}$. The number of pixels with $\boldsymbol{i}$ gray level is denoted as $\boldsymbol{f}$, and the occurence probability for image's $\boldsymbol{i}$ gray level is given by:

$$
p_{i}=\frac{f_{i}}{N}
$$


In two-level thresholding for an image (binarization), pixels are divided into two classes: $\boldsymbol{C}_{\boldsymbol{1}}$ with gray levels $[\mathbf{1}, \ldots, \boldsymbol{t}]$; and $\boldsymbol{C}_{\mathbf{2}}$ with gray levels $[\boldsymbol{t}+\mathbf{1}, \ldots, \boldsymbol{L}]$. Then the gray levels' probability distribution for two classes are:

Wherein:

$$
\begin{gathered}
C_{1}: \frac{p_{1}}{\omega_{1}(t)}, \ldots, \frac{p_{t}}{\omega_{1}(t)} \\
C_{2}: \frac{p_{t+1}}{\omega_{2}(t)}, \frac{p_{t+2}}{\omega_{2}(t)}, \ldots, \frac{p_{L}}{\omega_{2}(t)}
\end{gathered}
$$

$$
\omega_{1}(t)=\sum_{i=1}^{t} p_{i} \quad \omega_{2}(t)=\sum_{i=t+1}^{L} p_{i}
$$

Also, the average for $\boldsymbol{C}_{\boldsymbol{1}}$ and $\boldsymbol{C}_{2}$ classes is:

$$
\mu_{1}=\sum_{i=1}^{t} \frac{i \cdot p_{i}}{\omega_{1}(t)} \quad \mu_{2}=\sum_{i=t+1}^{L} \frac{i \cdot p_{i}}{\omega_{2}(t)}
$$

If $\boldsymbol{\mu}_{T}$ is the average intensity of the image it's easy to show that:

$$
\omega_{1} \cdot \mu_{1}+\omega_{2} \cdot \mu_{2}=\mu_{t} \quad \omega_{1}+\omega_{2}=1
$$

Using discriminant analysis, Otsu [35] defined the variance between thresholded image's classes as:

$$
\sigma_{B}^{2}=\omega_{1} \cdot\left(\mu_{1}-\mu_{t}\right)^{2}+\omega_{2} \cdot\left(\mu_{2}-\mu_{t}\right)^{2}
$$

For two-level thresholding, Otsu verified that the $t^{*}$ optimum threshold is chosen so that $\boldsymbol{\sigma}_{\boldsymbol{B}}^{2}$ is maximum [35]; that is:

$$
t^{*}=\max \left\{\sigma_{B}^{2}(t)\right\} \quad 1 \leq t<L
$$

Applying a threshold $\boldsymbol{t}$, the grayscale image, $\boldsymbol{f}(\boldsymbol{x}, \boldsymbol{y})$, will be binarized; labeling with ' 1 ' the pixels corresponding to the foreground and ' 0 ' are those of the background. Applying:

$$
g(x, y)=\left\{\begin{array}{l}
1<=>f(x, y)>t \\
0<=>f(x, y) \leq t
\end{array}\right.
$$

The main advantage of this method is that thresholding makes no initial assumption, since it assumes that the optimum threshold can be described only in terms of their means and variances. However its disadvantage is that it is used when there is a clear separation between classes observing the image's histogram. 


\subsection{Alpha-Beta Support Vector Associative Machine [34]}

An associative memory can be formulated, for operation as an input and output system, an idea that is outlined below:

$$
x \rightarrow M \rightarrow y
$$

Since the model of the $\alpha-\beta$ SVAM is autoassociative, we assume to have a pattern recognition problem, wherein the fundamental set has the form $\left\{\left(x^{\mu}, x^{\mu}\right) \mid \mu=1,2, \ldots, p\right\}$, with $x^{\mu} \in A^{n} \forall \mu \in\{1,2, \ldots, p\}$, where $n, p \in Z^{+}$and $A=\{0,1\}$.

The $x^{\mu}$ vectors are column vectors and the index of components is increasing from up to down, as illustrated bellow:

$$
x^{\mu}=\left(\begin{array}{c}
x^{\mu_{1}} \\
x^{\mu_{2}} \\
\cdots \\
x^{\mu_{n}}
\end{array}\right)
$$

One of the original ideas of the proposed model is trying to exploit the repeated information in the fundamental patterns. So, first a repeated pattern that contains information on all fundamental patterns is obtained; later this information is removed from the fundamental patterns, leaving only the information that differentiates a fundamental pattern of all others. This repeated information has inspired the new concept of support vector.

When an unknown pattern (can belong or not to the fundamental set) is presented to the model, which is intended to recognize with this model, we proceed to eliminate repeated information based on the pattern with this kind of information. This is simply the support vector for this fundamental set.

Now, we need to determine in some way, which of the fundamental patterns is less different with respect to this unknown pattern without repeated information (support vector). Is expected the less different fundamental pattern be the most similar. We just replace the missed information that was removed in the recovered fundamental pattern, and we have set the output pattern. We can see this method in Figures 1a and $1 \mathrm{~b}$. 


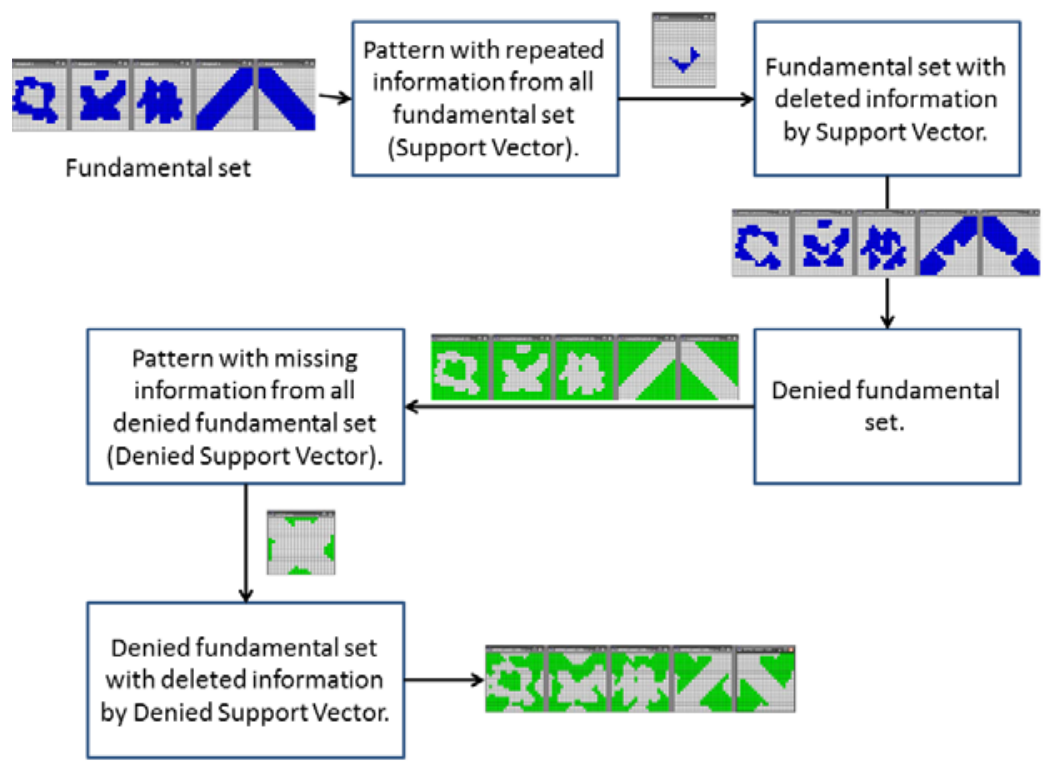

Fig. 1a. $\alpha-\beta$ SVAM Learning Diagram

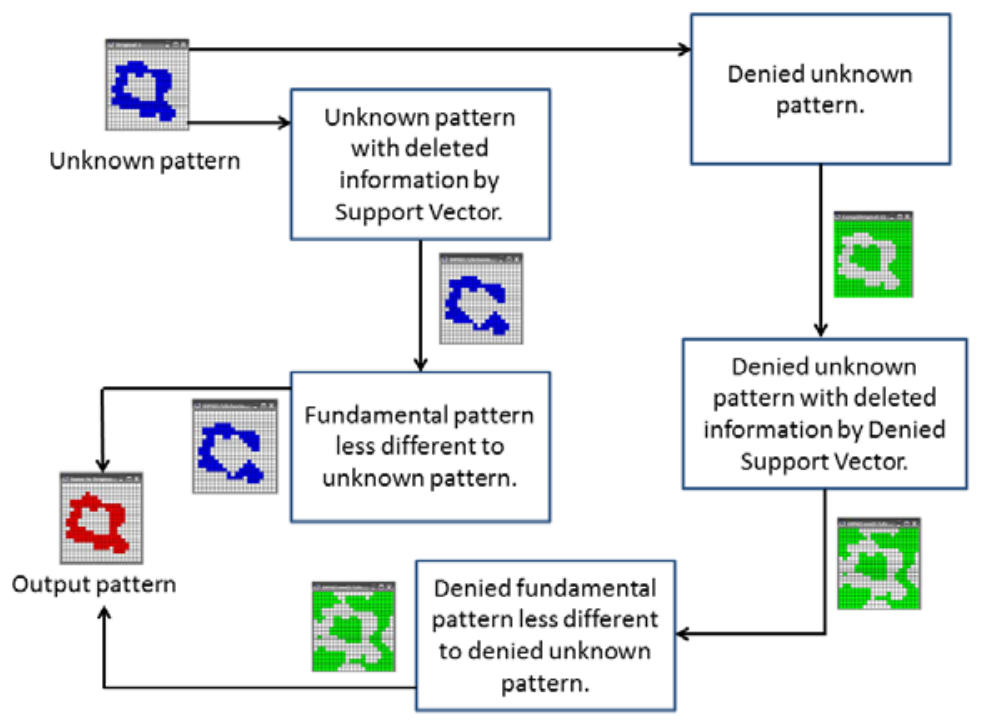

Fig. 1b. $\alpha-\beta$ SVAM Recall Diagram 


\section{Dataset}

For the development of this paper the image set we used grayscale mammograms of 259 patients between 35 and 60 years old. The image set is courtesy of Dr. Miguel Angel Guevara and co-authors, members of the Breast Cancer Digital Repository Consortium, University of Porto, in Portugal, among others [36].

Each image is a pattern that must be reduced for use in the $\alpha-\beta$ SVAM model, applying in pixels to retrieve and classify the image. This process was carried out in Matlab, implementation using Otsu method for each image binarizing and scale the image from $3300 \times 4080$ pixels to $330 \times 408$. We reduced the size image, but the new format used allows large amount of information is not lost in the binary pixel values.

The Figures $2 \mathrm{a}$ and $2 \mathrm{~b}$ show a mammography, first shows the original mammography, later we can see the binarized image applying Otsu method.

\section{Results}

To compare the accuracy of the proposed method in this work against other models conventionally used for cancer classification, the WEKA data mining and classification platform was used. In the particular case of the Multi Layer Perceptron neural network (MLP), the Matlab implementation was used instead of the one present in WEKA, given the improved memory usage characteristics offered by the former.

The $\alpha-\beta$ SVAM method was implemented with the Java language in the NetBeans Integrated Development Environment 7.4.

For validation purposes, the $\mathrm{k}$-fold cross validation technique was used, with $\mathrm{k}=10$ [37]. The experimental results exhibited by each model are shown in Table 1, while their corresponding confusion matrices are presented in Table 2. The metric used for comparison is overall accuracy, as seen in Table 1; and the confusion matrix allows us to review in detail the number of correctly cassified patterns for each case.

Table 1. Classification accuracy comparison.

\begin{tabular}{lll}
\hline Dataset & Algorithm & Accuracy (\%) \\
\hline Breast Cancer Digital & $\boldsymbol{\alpha - \beta}$ SVAM & $\mathbf{8 1 . 8 5}$ \\
Repository - D01 & k-NN & 80.31 \\
& Naïve Bayes & 76.83 \\
& SMO-SVM & 72.97 \\
& MLP & 70.27 \\
\hline
\end{tabular}




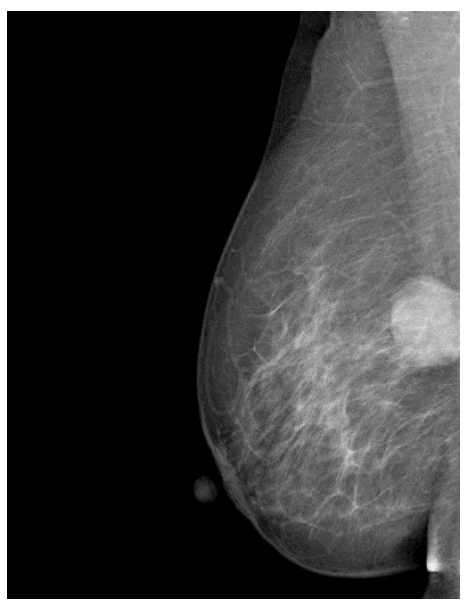

Fig. 2a. Original mammography (Source: [36])

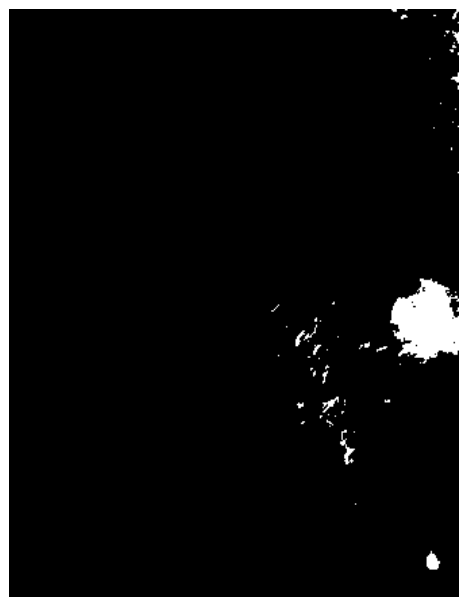

Fig. 2b. Binarized mammography by means of Otsu method

Table 2. Confusion matrix for each classifier.

\begin{tabular}{llcc}
\hline & & BENIGN & MALIGN \\
\hline BENIGN & $\boldsymbol{\alpha - \beta}$ SVAM & $\mathbf{1 3 1}$ & 16 \\
& k-NN & $\mathbf{1 3 1}$ & 16 \\
& Naïve Bayes & 115 & 32 \\
& SMO-SVM & 110 & 37 \\
& MLP & 112 & 35 \\
\hline MALIGN & $\alpha-\beta$ SVAM & 31 & 81 \\
& k-NN & 35 & 77 \\
& Naïve Bayes & 28 & $\mathbf{8 4}$ \\
& SMO-SVM & 33 & 79 \\
& MLP & 42 & 70 \\
\hline
\end{tabular}

\section{Conclusions}

Based on the results obtained, we can conclude about the model accuracy percentage, the more competitive is $\alpha-\beta$ SVAM with $81.85 \%$.

This proposal has shown being the best classification method compared to models with better global ranking for the breast cancer detection on the assumption that the cost of 
misclassified cases as benign and malignant is the same. In other situation we can see that the amount of well classified patterns as malignant cases is slightly greater using Naïve Bayes classifier.

The most practical method is proposed in this paper, being designed to use the image as a binary pattern classification, whose advantages against other classification models are:

* The $\alpha-\beta$ SVAM method does not require feature extraction or measurements on each mammogram obtained by a medical specialist.

* Not required a computational algorithm for feature selection, because $\alpha-\beta$ SVAM delete the repeated information in images.

\section{Acknowledgments}

The authors would like to thank the Instituto Politécnico Nacional (Secretaría Académica, COFAA, SIP, CIC, and CIDETEC), the CONACyT, and SNI for their economical support to develop this work.

\section{References}

1. NormaOficialMexicana, NOM-041-SSA2-2011, 2011. [Online]. In: http://dof.gob.mx/nota_detalle.php?codigo $=5194157 \&$ fecha $=09 / 06 / 2011$. [Accessed: 09-Nov-2013]

2. INSP: Un mes dedicado al cáncer de mama. 2013. In: http://www.insp.mx/avisos/2999-mes-cancermama.html. [Accessed: 20-Nov-2013]

3. IMSS: Guía de práctica clínica. Diagnóstico y Tratamiento del Cáncer de Mama. 2009. In: http://www.imss.gob.mx/profesionales/guiasclinicas/Documents/232GRR.pdf. [Accessed: 01-Dec2013]

4. J. Lopez Guerra, R. Gozalez, C. Parra, A. Martinez, A. Moreno, J. Peinado, V. Suarez, M. Cabeza, B. Quintana, M. Fernandez, and M. Ortiz: Application of artificial intelligence for breast cancer classification before radiatiotherapy. In: Reports Pract. Oncol. Radiother., vol. 18. April, pp. S391S392, Jun. 2013

5. V. L. Patel, E. H. Shortliffe, M. Stefanelli, P. Szolovits, M. R. Berthold, R. Bellazzi, and A. AbuHanna: The coming of age of artificial intelligence in medicine. In: Artif. Intell. Med., vol. 46, no. 1, pp. 5-17, May 2009

6. D. J. Rupert, L. B. Squiers, J. M. Renaud, N. S. Whitehead, R. J. Osborn, R. D. Furberg, C. M. Squire, and J. P. Tzeng: Communicating risk of hereditary breast and ovarian cancer with an interactive decision support tool. In: Patient Educ. Couns., vol. 92, no. 2, pp. 188-96, Aug. 2013

7. W. G. Harless, M. a Zier, M. G. Harless, R. C. Duncan, M. A. Braun, S. Willey, C. Isaacs, and R. D. Warren: Evaluation of a virtual dialogue method for breast cancer patient education. In: Patient Educ. Couns., vol. 76, no. 2, pp. 189-95, Aug. 2009

8. G. Valentini, R. Tagliaferri, and F. Masulli: Computational intelligence and machine learning in bioinformatics. In: Artif. Intell. Med., vol. 45, no. 2-3, pp. 91-6, 2009 
9. Y. Peng, Y. Zhang, and L. Wang: Artificial intelligence in biomedical engineering and informatics: an introduction and review. In: Artif. Intell. Med., vol. 48, no. 2-3, pp. 71-3, 2010

10. A. Yardimci: Soft computing in medicine. In: Appl. Soft Comput., vol. 9, no. 3, pp. 1029-1043, Jun. 2009

11. Han, S.: Associative memory in chronic schizophrenia: a computational model. In: Schizophr. Res., vol. 61 , no. 2-3, pp. 255-263, Jun. 2003

12. D. Gil, M. Johnsson, J. M. Garcia Chamizo, A. S. Paya, and D. R. Fernandez: Application of artificial neural networks in the diagnosis of urological dysfunctions. Expert Syst. Appl., vol. 36, no. 3, pp. 5754-5760, Apr. 2009

13. D. Gil, J. L. Girela, J. De Juan, M. J. Gomez-Torres, and M. Johnsson: Predicting seminal quality with artificial intelligence methods. Expert Syst. Appl., vol. 39, no. 16, pp. 12564-12573, Nov. 2012

14. J. M. Tenório, A. D. Hummel, F. M. Cohrs, V. L. Sdepanian, I. T. Pisa, and H. de Fátima Marin: Artificial intelligence techniques applied to the development of a decision-support system for diagnosing celiac disease. In: Int. J. Med. Inform., vol. 80, no. 11, pp. 793-802, Nov. 2011

15. A. Marcano-Cedeño, P. Chausa, A. García, C. Cáceres, J. M. Tormos, and E. J. Gómez: Artificial metaplasticity prediction model for cognitive rehabilitation outcome in acquired brain injury patients. In: Artif. Intell. Med., vol. 58, no. 2, pp. 91-9, Jun. 2013

16. B. Samanta, G. L. Bird, M. Kuijpers, R. Zimmerman, G. P. Jarvik, G. Wernovsky, R. R. Clancy, D. J. Licht, J. W. Gaynor, and C. Nataraj: Prediction of periventricular leukomalacia. Part II: Selection of hemodynamic features using computational intelligence. Artif. Intell. Med., vol. 46, no. 3, pp. 217-31, Jul. 2009

17. C.-L. Chang and M.-Y. Hsu: The study that applies artificial intelligence and logistic regression for assistance in differential diagnostic of pancreatic cancer. In: Expert Syst. Appl., vol. 36, no. 7, pp. 10663-10672, Sep. 2009

18. W. Zhao and C. E. Davis: A modified artificial immune system based pattern recognition approach an application to clinical diagnostics. In: Artif. Intell. Med., vol. 52, no. 1, pp. 1-9, May 2011

19. A. Keleş, A. Keleş, and U. Yavuz: Expert system based on neuro-fuzzy rules for diagnosis breast cancer. In: Expert Syst. Appl., vol. 38, no. 5, pp. 5719-5726, May 2011

20. J. Nahar, T. Imam, K. S. Tickle, B. M. Shawkat Ali, and Y.-P. P. Chen: Computational intelligence for microarray data and biomedical image analysis for the early diagnosis of breast cancer. Expert Syst. Appl., vol. 39, no. 16, pp. 12371-12377, Nov. 2012

21. J. M. Jerez, I. Molina, P. J. García-Laencina, E. Alba, N. Ribelles, M. Martín, and L. Franco: Missing data imputation using statistical and machine learning methods in a real breast cancer problem. Artif. Intell. Med., vol. 50, no. 2, pp. 105-15, Oct. 2010

22. L. Álvarez Menéndez, F. J. de Cos Juez, F. Sánchez Lasheras, and J. Álvarez Riesgo: Artificial neural networks applied to cancer detection in a breast screening programme. Math. Comput. Model., vol. 52, no. 7-8, pp. 983-991, Oct. 2010

23. Akay, M. F.: Support vector machines combined with feature selection for breast cancer diagnosis. In: Expert Syst. Appl., vol. 36, no. 2, pp. 3240-3247, Mar. 2009

24. H.-L. Chen, B. Yang, J. Liu, and D.-Y. Liu: A support vector machine classifier with rough set-based feature selection for breast cancer diagnosis. In: Expert Syst. Appl., vol. 38, no. 7, pp. 9014-9022, Jul. 2011

25. B. Zheng, S. W. Yoon, and S. S. Lam: Breast cancer diagnosis based on feature extraction using a hybrid of K-means and support vector machine algorithms. In: Expert Syst. Appl., vol. 41, no. 4, pp. 1476-1482, Mar. 2014 
26. J. W. F. Catto, M. F. Abbod, P. J. Wild, D. Linkens, C. Pilarsky, I. Rehman, D. J. Rosario, S. Denzinger, M. Burger, R. Stoehr, R. Knuechel, A. Hartmann, and F. C. Hamdy: The application of artificial intelligence to microarray data: identification of a novel gene signature to identify bladder cancer progression. In: Eur. Urol., vol. 57, no. 3, pp. 398-406, Mar. 2010

27. A. Marcano-Cedeño, J. Quintanilla-Domínguez, and D. Andina: Breast cancer classification applying artificial metaplasticity algorithm. In: Neurocomputing, vol. 74, no. 8, pp. 1243-1250, Mar. 2011

28. A. Marcano-Cedeño, J. Quintanilla-Domínguez, and D. Andina: WBCD breast cancer database classification applying artificial metaplasticity neural network. In: Expert Syst. Appl., vol. 38, no. 8, pp. 9573-9579, Aug. 2011

29. M. Karabatak and M. C. Ince: An expert system for detection of breast cancer based on association rules and neural network. In: Expert Syst. Appl., vol. 36, no. 2, pp. 3465-3469, Mar. 2009

30. K. Park, A. Ali, D. Kim, Y. An, M. Kim, and H. Shin: Robust predictive model for evaluating breast cancer survivability. In: Eng. Appl. Artif. Intell., vol. 26, no. 9, pp. 2194-2205, Oct. 2013

31. N. Cruz-Ramírez, H.-G. Acosta-Mesa, H. Carrillo-Calvet, and R.-E. Barrientos-Martínez: Discovering interobserver variability in the cytodiagnosis of breast cancer using decision trees and Bayesian networks. In: Appl. Soft Comput., vol. 9, no. 4, pp. 1331-1342, Sep. 2009

32. Yáñez-Márquez, C.: Memorias Asociativas Basadas en Relaciones de Orden y Operadores Binarios. CIC IPN, México, 2002

33. R. Santiago-Montero: Clasificador híbrido de patrones basado en la Lernmatrix de Steinbuch y el Linear Associator de Anderson-Kohonen. CIC IPN, México, 2003

34. López-Leyva, L. O.: Máquinas Asociativas Alfa-Beta con Soporte Vectorial, CIC IPN, México, 2008

35. UNQ: Segmentación por Umbralización. $2005 . \quad$ In: http://iaci.unq.edu.ar/materias/vision/archivos/apuntes/Segmentaci\%C3\%B3n\%20por\%20umbralizac i\%C3\%B3n\%20-\%20M\%C3\%A9todo\%20de\%20Otsu.pdf. [Accessed: 20-Nov-2013]

36. M. A. Guevara Lopez and B. C. D. R. Consortium: Breast Cancer Digital Repository. University of Porto, Porto, Portugal, 2012

37. R. Duda, P. Hart and D. Stork: Pattern Classification. 2nd ed. New York, USA: Wiley Interscience, 2000, pp. 483-485 
